\title{
CESAREAN DELIVERY IN PREGNANCIES OF MOTHERS WITH SYSTEMIC LUPUS ERYTHEMATOSUS IN A TERTIARY HOSPITAL
}

\author{
Juliana Bühring ${ }^{1, *}$, Sergio Henrique Oliveira dos Santos ${ }^{1}$, Bárbara Seabra Carneiro으. Domingos Sávio Nunes de Lima² \\ 1. Hospital Universitário Getúlio Vargas, Manaus (AM), Brazil. 2. Universidade Federal do Amazonas, Manaus (AM), Brazil. \\ *Corresponding author: jubuhring@hotmail.com
}

\section{BACKGROUND}

Systemic lupus erythematosus (SLE) is an autoimmune disease that has a variable clinical picture and a remitting-recurrent character. It can reach a female:male ratio 9:1, affecting negatively maternal reproductive aspect, as disease exacerbation occurs in about $50 \%$ of pregnancies. Some studies point out to an increased frequency of cesarean section in SLE pregnant. In Brazil, from 2003 to 2018, the rate of cesarean delivery was about the same vaginal one. The aims of the present study were to describe the frequency of cesarean section in SLE mothers, to compare it with general population frequency, and to verify if there is a significant difference between proportion of cesarean section in mothers with active disease and those without it.

\section{MATERIALS AND METHODS}

A descriptive cross-sectional study was performed in a rheumatology service of a tertiary hospital that follows about 800 patients with SLE being approximately 700 female patients. The patients who had at least one pregnancy from January 2003 until December 2019, were in regular follow-up and had a born alive infant were included. Patients unable to provide data about the delivery type were excluded; categorical variable was correlated using the Fisher's exact test and the proportions were tested using the binomial test.

\section{RESULTS}

Seventy-seven patients were included with 93 pregnancies; the mean age at pregnancy was 27.9 years, standard deviation (SD) 5.5 years. The mean disease length was 6.0 years SD 4.15 years. There were 62 cesarean deliveries (66.7\%); when compared with general population rates, there was higher cesarean deliveries in SLE mothers $(p=0.001, \mathrm{OR}=1.97,95 \% \mathrm{Cl}=1.64-2.36)$. From 50 pregnancies where mother had active disease (AD), 36 undergone cesarean delivery $(72.0 \%)$ and from group where there was no disease activity $(n=43), 26$ cesarean section was performed $(60.5 \%)$.

After comparing these two groups, there was difference of about $11.5 \%$ more cesarean section in AD group; however, this difference was not significant ( $p=0.275)$, and there was no significant association between disease activity and cesarean section $(p=0.275, \mathrm{OR}=0.595,95 \% \mathrm{Cl}=0.249-1.42)$. (Fig. 1 and Table 1 ).

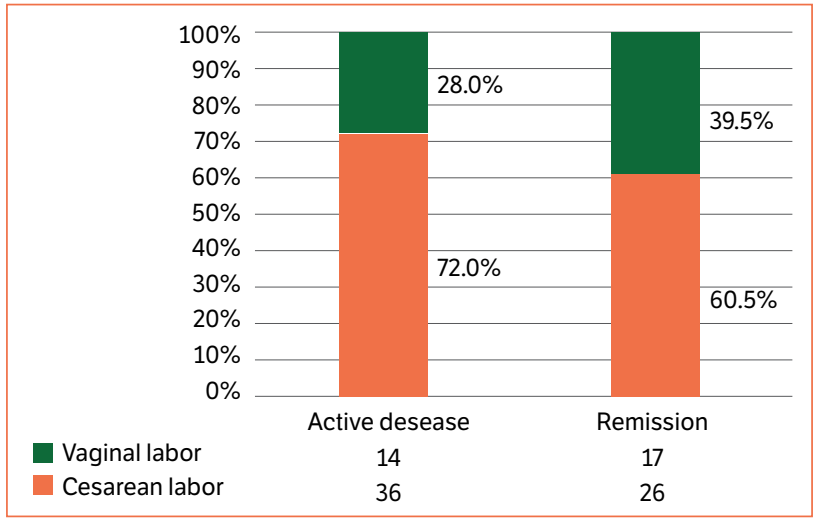

Figure 1. Distribution of frequency of delivery route by disease state. 
Table 1. Z-score of difference between delivery route and disease activity state.

\begin{tabular}{ccccccccc}
\hline \multirow{2}{*}{ Delivery route } & \multicolumn{2}{c}{ Active disease } & \multicolumn{2}{c}{ Remission } & \multicolumn{3}{c}{ Difference } \\
\cline { 2 - 10 } & $\mathrm{n}$ & $\%$ & $\mathrm{n}$ & $\%$ & $\mathrm{n}$ & $\%$ & $\mathrm{Z}$ & $\mathrm{P}$ \\
\hline Vaginal & 14 & 28 & 17 & 39.5 & -3 & -13.9 & -1.41 & 0.192 \\
\hline Cesarean & 36 & 72 & 26 & 60.5 & 14 & 11.5 & 1.18 & 0.275 \\
\hline
\end{tabular}

\section{CONCLUSION}

In our study, we could observe a significant higher proportion of cesarean delivery than in general population; we also observed a higher, but not significant proportion of cesarean section in patients who experienced disease activity during pregnancy. The factors accountable by these findings in our sample need to be better elucidated in future studies. 